\title{
Editorial
}

\section{Nanomaterials for Medical Applications: Benefits and Risks}

\author{
Ecaterina Andronescu, ${ }^{1}$ Jared M. Brown, ${ }^{2}$ Faik Nuzhet Oktar, ${ }^{3}$ \\ Simeon Agathopoulos, ${ }^{4}$ Joshua Chou, ${ }^{5}$ and Akiko Obata ${ }^{6}$ \\ ${ }^{1}$ Department of Science and Engineering of Oxide Materials and Nanomaterials, Faculty of Applied Chemistry and Materials Science, \\ University Politehnica of Bucharest, Bucharest, Romania \\ ${ }^{2}$ Department of Pharmaceutical Sciences, Skaggs School of Pharmacy, University of Colorado Anschutz Medical Campus, \\ Aurora, CO, USA \\ ${ }^{3}$ Bioengineering Department, Faculty of Engineering and Advanced Nanomaterials Research Laboratory, \\ Marmara University, Istanbul, Turkey \\ ${ }^{4}$ Materials Science and Engineering Department, Ioannina University, Ioannina, Greece \\ ${ }^{5}$ Advanced Tissue Regeneration \& Drug Delivery Group, School of Medical and Molecular Biosciences, \\ University of Technology, Sydney, Australia \\ ${ }^{6}$ Graduate School of Engineering, Nagoya Institute of Technology, Nagoya, Japan
}

Correspondence should be addressed to Ecaterina Andronescu; ecaterina.andronescu@upb.ro

Received 16 December 2015; Accepted 16 December 2015

Copyright (C) 2016 Ecaterina Andronescu et al. This is an open access article distributed under the Creative Commons Attribution License, which permits unrestricted use, distribution, and reproduction in any medium, provided the original work is properly cited.

Since their discovery, nanomaterials have been intensively studied for both industrial and medical applications. For the medical sector, one important aspect, which is being increasingly recognized, is the safety concerns related to the handling and use of nanomaterials. Currently, scientists are trying to determine whether the benefits outweigh the risks.

This special issue is structured in several subtopics covering: carbon based materials alone or in association with other nanostructured components; various nanoparticles, including quantum dots; or composite nanomaterials as regenerative or drug delivery systems.

In the field of carbon based materials, two very hot subjects are discussed, namely, the influence of nitrogendoped multiwall carbon nanotubes on the proliferation of fibroblasts and the possibility of the use of Pt nanoparticle/graphene composite materials as electrochemical sensor of hydrogen peroxide. The efficiency of the Pt/graphenemodified glassy carbon (GC) electrodes was characterized by cyclic voltammetry, the main difference between the samples being the size of the nanoparticles $(1.3 ; 1.7 ; 2.9$; and $4.3 \mathrm{~nm}$ ). The authors found that best results (highest reduction current, the best detection limit, and the best sensitivity) are obtained for the electrodes containing
$1.7 \mathrm{~nm}$ Pt nanoparticles. Nitrogen-doped multiwall carbon nanotubes were found to be well tolerated below $7 \mu \mathrm{g} / \mathrm{mL}$ and consequently are suggested by the authors as safe for use in biomedical applications below certain concentrations. Also, this work highlighted that cytotoxicity is dependent on concentration and exhibits a higher toxicity on enzymatic stressed cells than on the nonstressed ones.

The risk evaluation of different nanoparticles is currently of interest worldwide. Silver and magnetite nanoparticles are also studied in this special issue highlighting the advances from the point of view of application and toxicity. Silver, for instance, was studied from the point of view of risk evaluation of silver nanoparticles exposure from sprays and it was found that, depending on exposure type, the limit of exposure ranges between 59 and 146 for inhalation, much lower than 1000 which means the no-risk level while the dermal exposure risk was between 20000 and $500000(\sim 2-50 \mu \mathrm{g} / \mathrm{kg} /$ day $)$. So, the exposure of people to silver containing aerosols is risky. Silver and magnetite nanoparticles were also studied from the point of view of the impact of exposure of these nanoparticles to cholesterol uptake by macrophages. This paper clearly indicates that long-term impact of nanoparticles on cellular function must be taken into account before using 
nanoparticles for biomedical applications. Lipophilic bismuth dimercaptopropanol nanoparticles have a very important antimicrobial activity, but at this moment there are no sufficient data. Based on these results, these nanoparticles at a level of up to $100 \mu \mathrm{M}$ do not cause damage to blood cells. CdTe quantum dots are good candidates for bioimaging but the evaluation of the toxicity must be done in order to avoid any risks. Based on a newly developed evaluation protocol based on protein expression, it was found that the quantum dot shell plays an important role due to first direct contact with cells. The CdSeS nanostructures were analyzed from the point of view of the correlation between synthesis route and applications, especially based on the toxicity of these nanoparticles depending on the synthesis route and used precursors and solvents. Based on this study, such safe, economic, environment friendly, and suitable for largescale production of alloyed CdSeS nanostructures with high photoluminescence, high stability, and low/no cytotoxicity are still much desired.

The role of nanocomposites for biomedical applications is also discussed in this special issue. The safe use of nanocomposite bone material was examined in a study of a thirty-one-patient clinical trial. The nanocomposite bone material was ultimately found to be useful in the field of orthopedics. Engineered materials can be also improved by adding beneficial components and consequently new functionalities. In this special issue, novel tetracycline loaded collagen/hydroxyapatite composite materials are presented for use in nanomedicine.

Overall, nanomaterials are expected to solve challenges in the health sector through biosensors, contrast agents, and targeted drug vehicles that can deliver antimicrobial or antitumoral agents. Unfortunately, the long-term implications are mainly unknown. However, there are some nanomaterials which already have demonstrated adverse behavior in biological systems. This special issue provides a comprehensive update in the field of nanomaterials, especially highlighting the benefits and risks related to use of nanomaterials.

\section{Ecaterina Andronescu Jared M. Brown Faik Nuzhet Oktar Simeon Agathopoulos Joshua Chou Akiko Obata}



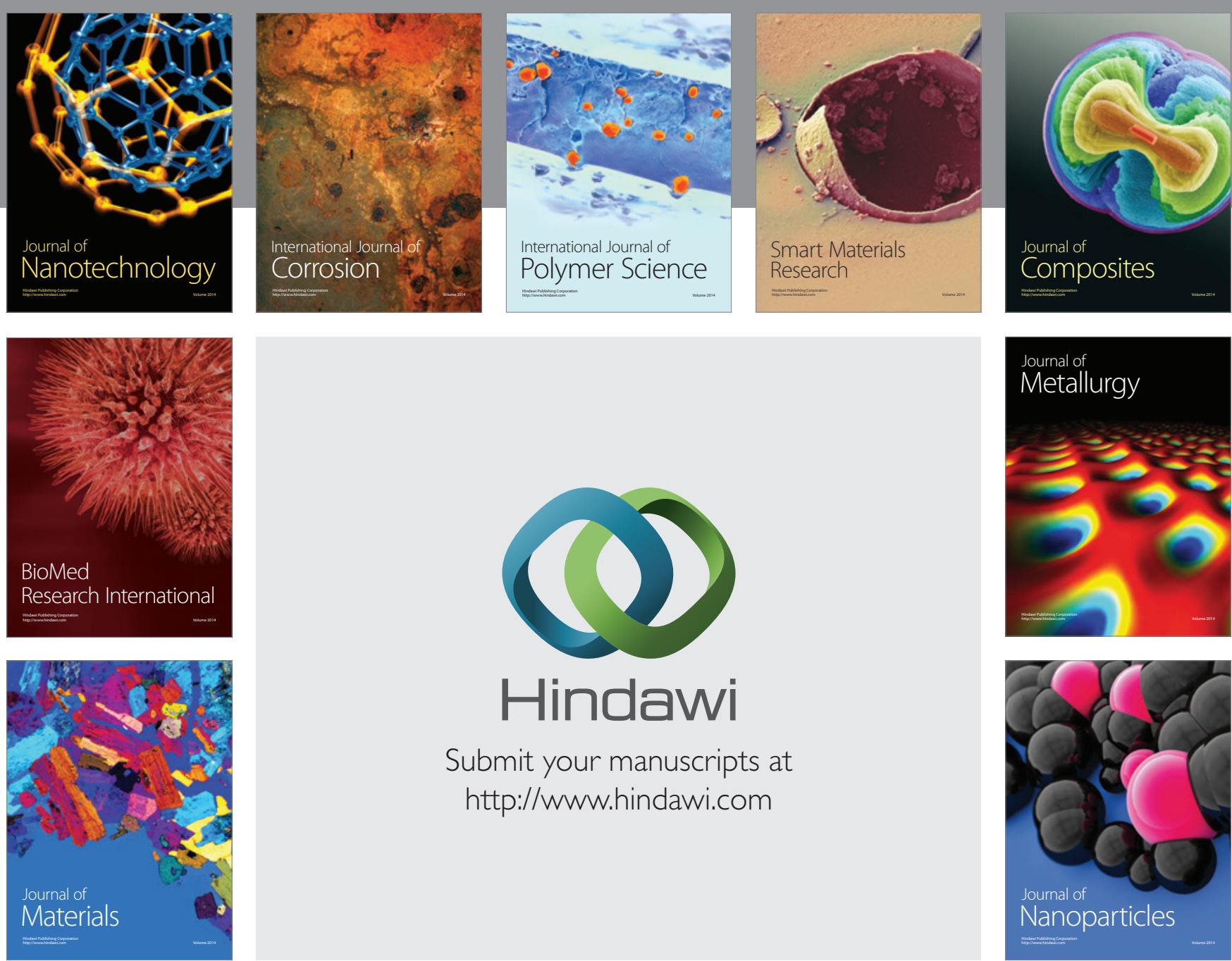

\section{Hindawi}

Submit your manuscripts at

http://www.hindawi.com

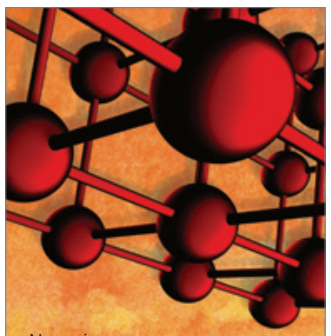

Materials Science and Engineering
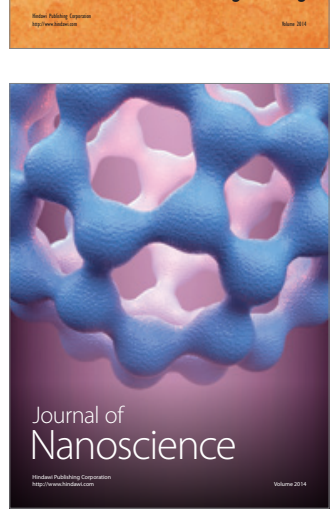
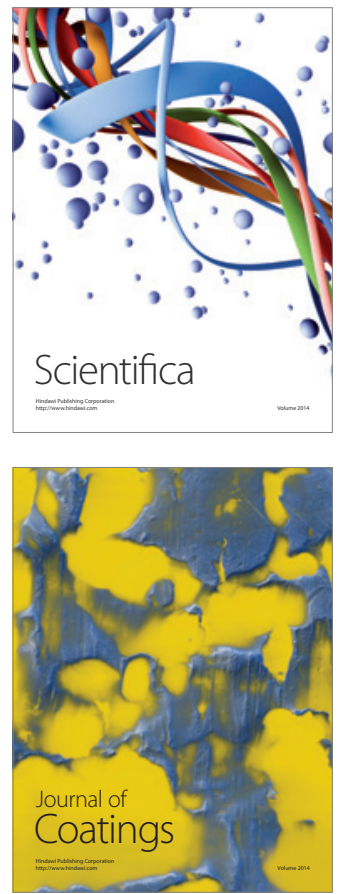
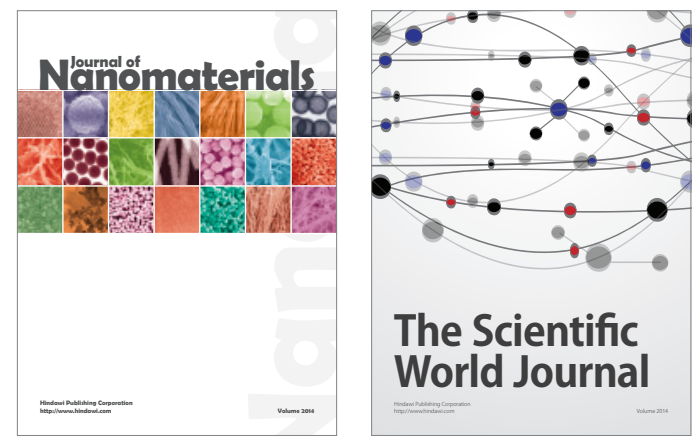

The Scientific World Journal
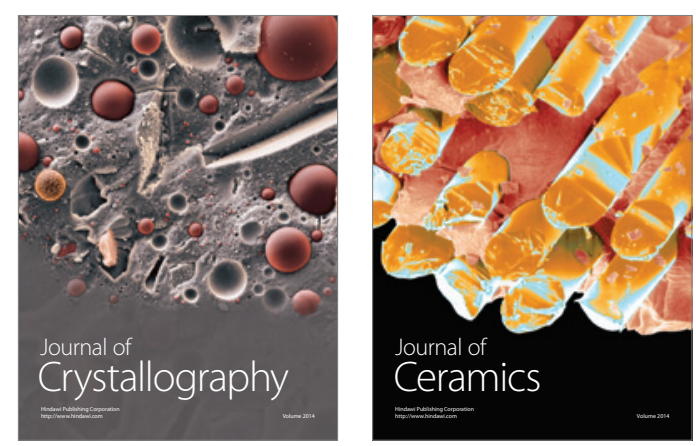
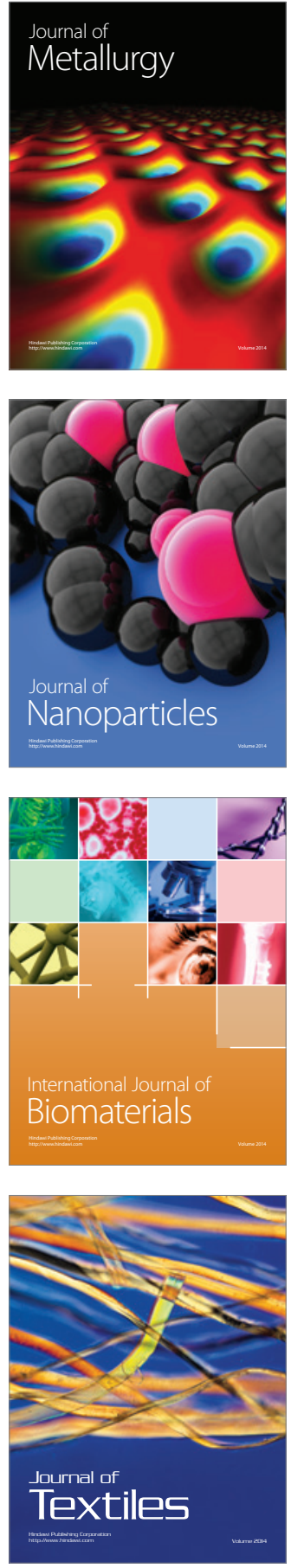